\title{
Article \\ Humidity Sensitivity of Hydration of Expansive Agent and Its Expansive Efficiency in Ultra-High Performance Concrete
}

\author{
Yujiang Wang ${ }^{1,2}, *$, Qian Tian ${ }^{2}$, Hua $\mathrm{Li}^{1,2}$ and Yang Wang ${ }^{2}$ \\ 1 Jiangsu Key Laboratory of Construction Materials, College of Materials Science and Engineering, \\ Southeast University, Nanjing 211189, China; lihua@cnjsjk.cn \\ 2 State Key Laboratory of High Performance Civil Engineering Materials, Jiangsu Sobute New \\ Materials Co., Ltd., Nanjing 211103, China; tianqian@cnjsjk.cn (Q.T.); wangyang@cnjsjk.cn (Y.W.) \\ * Correspondence: wangyujiang@cnjsjk.cn
}

check for updates

Citation: Wang, Y.; Tian, Q.; Li, H.; Wang, Y. Humidity Sensitivity of Hydration of Expansive Agent and Its Expansive Efficiency in Ultra-High Performance Concrete. Crystals 2022, 12, 195. https://doi.org/10.3390/ cryst12020195

Academic Editors: Shunbo Zhao, Juntao Ma, Shan Li and

Shujun Zhang

Received: 31 December 2021

Accepted: 26 January 2022

Published: 28 January 2022

Publisher's Note: MDPI stays neutral with regard to jurisdictional claims in published maps and institutional affiliations.

Copyright: (c) 2022 by the authors. Licensee MDPI, Basel, Switzerland. This article is an open access article distributed under the terms and conditions of the Creative Commons Attribution (CC BY) license (https:/ / creativecommons.org/licenses/by/ $4.0 /)$.

\begin{abstract}
Ultra-high performance concrete (UHPC) has a potential cracking risk due to its large autogenous shrinkage. The use of an expansive agent is an effective approach to reduce autogenous shrinkage of UHPC. However, different kinds of expansive agents show different expansive efficiency in UHPC. To study the cause for the difference in expansive efficiency, this study selected three expansive agents, namely highly reactive $\mathrm{MgO}$-based, medium reactive $\mathrm{MgO}$-based, and $\mathrm{CaO}$-based expansive agents, and carried out the following experiments: autogenous shrinkage, hydration heat, hydration process of expansive agent under different relative humidity $(\mathrm{RH})$, and micrographs. The results showed that the $\mathrm{CaO}$-based expansive agent has high hydration activity at $\mathrm{RH}$ of more than $44.0 \%$, while the hydration activity of two kinds of $\mathrm{MgO}$-based expansive agents, especially a medium reactive $\mathrm{MgO}$-based expansive agent, decreases significantly when $\mathrm{RH}$ drops below $80 \%$. Meanwhile, the CaO-based expansive agent had higher expansive efficiency in UHPC than the MgObased expansive agent. This study suggested that the $\mathrm{CaO}$-based expansive agent is more suitable for compensating autogenous shrinkage of UHPC due to its low humidity sensitivity compared to the MgO-based expansive agent.
\end{abstract}

Keywords: ultra-high performance concrete; autogenous shrinkage; hydration; CaO-based expansive agent; $\mathrm{MgO}$-based expansive agent

\section{Introduction}

Ultra-high performance concrete (UHPC) is a kind of cement-based composite with excellent properties such as ultra-high compressive strength $(\geq 120 \mathrm{MPa})$ and extremely low porosity [1-3]. However, UHPC has large early age shrinkage, especially autogenous shrinkage, due to factors such as extremely low water to binder ratio ( $w / b$, typically $\leq 0.20)$, incorporation of ultra-fine powder, and almost no coarse aggregate [2,3]. Due to the extremely low $\mathrm{w} / \mathrm{b}$ of UHPC, the mixing water is insufficient for the hydration of cementitious materials. The increase of capillary tension in pores due to the decrease of internal relative humidity (RH) is the main driving force that results in autogenous shrinkage of UHPC. Recently, studies have shown that autogenous shrinkage of UHPC can reach approximately $1000 \mu \varepsilon[4,5]$. When this large shrinkage is constrained, cracks may occur and lead to negative impacts on the durability and safety of UHPC structures [6].

Much work so far has focused on mitigation of autogenous shrinkage of UHPC [7]. Several approaches have been developed, such as incorporation of Supplementary Cementitious Materials [8], the use of shrinkage reducing and expansive agents [9-12], internal curing by a superabsorbent polymer $[13,14]$, and increase of particle size and volume ratio of aggregates [15-17]. Furthermore, recent studies have shown that the application of nanomaterials can effectively reduce the autogenous shrinkage, and improve the rheology and hydration properties of UHPC [18-20]. Using expansive agents to compensate shrinkage of concrete is an effective approach in concrete engineering. Three types of 
expansive agents, namely sulphoaluminate type, $\mathrm{CaO}$-based, and $\mathrm{MgO}$-based expansive agents, are widely used. However, previous studies have suggested that a sulphoaluminate type agent is not suitable for UHPC due to its high water requirement in hydration process and unstable expansion product $[7,21]$. At present, $\mathrm{MgO}$-based and $\mathrm{CaO}-\mathrm{based}$ expansive agents have been intensively investigated by researchers and have been proved to reduce the autogenous shrinkage of UHPC effectively. Li et al. [11] studied the effects of different reactive $\mathrm{MgO}$-based expansive agents on the autogenous shrinkage of UHPC, and suggested that the addition of $6 \%$ highly reactive $\mathrm{MgO}$-based expansive agent could effectively compensate the shrinkage of UHPC. The studies of Shen et al. [10] showed that the addition of a calcium-sulfoaluminates-CaO-based expansive agent could mitigate the shrinkage of UHPC, but the efficiency was not high. The hydration products of MgO-based and $\mathrm{CaO}-$ based expansive agents are mainly $\mathrm{Mg}(\mathrm{OH})_{2}$ and $\mathrm{Ca}(\mathrm{OH})_{2}$, respectively. The volume increase of the expansive agent in the hydration process is the main mechanism for shrinkage compensation of concrete. Most of the studies have focused on the hydration degree, micrographs of hydration products, and temperature sensitivity of the expansive agents $[10,11,22,23]$. It should be noted that internal RH of UHPC decreases fast and can reach a lower RH compared with ordinary and high-strength concrete [24,25]. Consequently, internal RH of UHPC is an important factor affecting the hydration and expansive efficiency of the expansive agents. However, there are few reports about this factor.

This study focuses on the humidity sensitivity of the hydration of expansive agent (i.e., hydration activity under different RH levels) to study mechanisms for expansive efficiency of expansive agent in UHPC. Three kind of expansive agents of highly reactive MgO-based, medium reactive $\mathrm{MgO}$-based and $\mathrm{CaO}$-based expansive agents were selected in this study. It should be noted that the three kinds of expansive agents, especially medium reactive $\mathrm{MgO}-$ based and $\mathrm{CaO}-$ based expansive agents, are widely used in concrete engineering in China $[19,26,27]$. This research will provide an effective method for the selection of expansive agent in preparation of UHPC.

\section{Materials and Methods}

\subsection{Materials}

Portland cement (52.5 grade) and silica fume were used in this study. Their chemical compositions are shown in Table 1 . River sand with apparent density of $2640 \mathrm{~kg} / \mathrm{m}^{3}$ and fineness modulus of 2.60 was used as fine aggregate. Basalt crushed stone with apparent density of $2800 \mathrm{~kg} / \mathrm{m}^{3}$ and particle size of $5-10 \mathrm{~mm}$ was used as coarse aggregate. Magnesite produced in Haicheng, Liaoning Province, is used as raw material for preparing $\mathrm{MgO}-$ based expansive agent. Magnesite was first crushed into fine particles less than $2 \mathrm{~mm}$. Then, magnesite powder is calcined in electric furnace at $750-850{ }^{\circ} \mathrm{C}$ for $1 \mathrm{~h}$ to prepare highly reactive $\mathrm{MgO}$-based expansive agent (H-MEA), and calcined at $950-1050{ }^{\circ} \mathrm{C}$ for $1 \mathrm{~h}$ to prepare medium reactive MgO-based expansive agent (M-MEA). CaO-based expansive agent (CA) was prepared by grinding expansive clinker to an average particle size of $45 \pm 5 \mu \mathrm{m}$. The clinker is provided by Jiangsu Sobute New Material Co., Ltd. (see Figure 1). The chemical composition of $\mathrm{CaO}$-based and $\mathrm{MgO}$-based expansive agents are shown in Table 1.

Table 1. Chemical compositions of the materials (\%).

\begin{tabular}{cccccccc}
\hline & $\mathbf{C a O}$ & $\mathbf{M g O}$ & $\mathbf{F e}_{\mathbf{2}} \mathbf{O}_{\mathbf{3}}$ & $\mathbf{A l}_{\mathbf{2}} \mathbf{O}_{\mathbf{3}}$ & $\mathbf{S i O}_{\mathbf{2}}$ & $\mathbf{S O}_{\mathbf{3}}$ & $\mathbf{L}$ \\
\hline Cement & 64.21 & 1.55 & 3.94 & 4.29 & 19.89 & 3.25 & $/$ \\
Silica fume & 0.56 & 2.10 & 0.75 & 0.06 & 93.55 & $/$ & 2.31 \\
H-MEA & 1.78 & 94.8 & 0.93 & 0.18 & 0.52 & 1.76 \\
M-MEA & 1.86 & 95.8 & 1.02 & 0.17 & 0.54 & 1 \\
CA & 81.9 & 1.5 & 0.23 & 3.33 & 1.18 & 10.40 & 1.35 \\
\hline
\end{tabular}




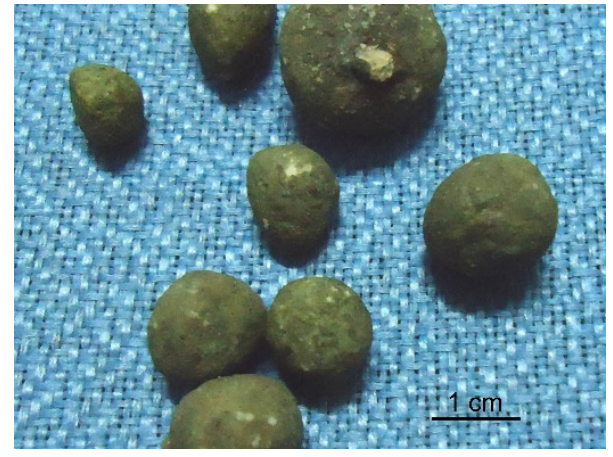

Figure 1. Clinker of CaO-based expansive agent.

The mix proportions of UHPC studied are shown in Table 2. All concrete mixtures have a $0.17 \mathrm{w} / \mathrm{b}$, which is widely used in concrete engineering. UHPC is mixed with coarse aggregate to reduce its autogenous shrinkage. The dosage of $\mathrm{MgO}$-based expansive agent is $4 \%$ and $6 \%$ of the mass of cementitious material, while the dosage of CaO-based expansive agent is $1 \%, 2 \%$ and $3 \%$. A commercial powder polycarboxylate-based superplasticizer was used for all mixtures. The dosage of the superplasticizer is $1.1 \%$ of the mass of cementitious materials. It should be noted that fiber is not considered in UHPC mix proportion, in order to avoid the influence of fiber on autogenous shrinkage.

Table 2. Mix proportions of UHPC $\left(\mathrm{kg} / \mathrm{m}^{3}\right)$.

\begin{tabular}{cccccccccc}
\hline & Water & Cement & $\begin{array}{c}\text { Silica } \\
\text { Fume }\end{array}$ & $\begin{array}{c}\text { Fine } \\
\text { Aggregate }\end{array}$ & $\begin{array}{c}\text { Coarse } \\
\text { Aggregate }\end{array}$ & H-MEA & M-MEA & CA & Superplasticizer \\
\hline Control & 201 & 1125 & 59 & 592 & 480 & $/$ & $/$ & $/$ & 13 \\
H-MEA-4\% & 201 & 1080 & 57 & 592 & 480 & 47 & $/$ & $/$ & 13 \\
H-MEA-6\% & 201 & 1057 & 56 & 592 & 480 & 71 & $/$ & $/$ & 13 \\
M-MEA-4\% & 201 & 1080 & 57 & 592 & 480 & $/$ & 47 & $/$ & 13 \\
M-MEA-6\% & 201 & 1057 & 56 & 592 & 480 & $/$ & 71 & $/$ & 13 \\
CA-1\% & 201 & 1114 & 59 & 592 & 480 & $/$ & $/$ & 13 \\
CA-2\% & 201 & 1102 & 58 & 592 & 480 & $/$ & $/$ & 24 & 13 \\
CA-3\% & 201 & 1091 & 57 & 592 & 480 & $/$ & $/$ & 36 & 13 \\
\hline
\end{tabular}

\subsection{Autogenous Shrinkage}

Figure 2 shows the apparatus for measuring autogenous shrinkage of UHPC. Concrete mixture was first cast into a cylindrical PVC tube $(\varnothing 100 \mathrm{~mm} \times 500 \mathrm{~mm})$ pre-sealed by epoxy resin at the bottom. Then, the concrete surface was covered with plastic film. When the concrete loses fluidity, a metal head was embedded in the surface concrete. After that, the surface of the specimen is covered with paraffin to prevent water evaporation. The vertical autogenous deformation was measured by a dial gauge at different ages. The test zero point is the initial setting of concrete, which was determined by penetration resistance according to ASTM C403 [28]. The ambient temperature of the test was $20.0 \pm 1.0{ }^{\circ} \mathrm{C}$.

\subsection{Internal Relative Humidity}

Internal RH of UHPC was measured using a humidity sensor (HC2-S, Rotronic, Bassersdorf, Switzerland) with an accuracy of $0.8 \%$ RH. Test methods referred to literature [13], and the test procedure was as follows: UHPC mixture was first placed in a sealed plastic bottle. At the age of $10 \mathrm{~h}$, the UHPC sample was crushed into pieces. Then, 2.36-4.74 $\mathrm{mm}$ pieces were chosen by sieves and inserted into a metal chamber for $\mathrm{RH}$ measurement. In addition, the metal chamber was placed in a water bath to keep the temperature of $20 \pm 0.1^{\circ} \mathrm{C}$. 


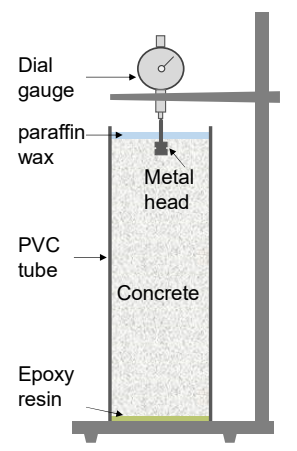

Figure 2. Apparatus for autogenous shrinkage measurement.

\subsection{Hydration of Expansive Agent under Different $R H$}

As shown in Figure 3, the relative humidity of $93 \%, 81 \%$ and $44 \%$ at $20{ }^{\circ} \mathrm{C}$ is achieved through three saturated salt solutions of $\mathrm{KNO}_{3},(\mathrm{NH} 4)_{2} \mathrm{SO}_{4}$, and $\mathrm{K}_{2} \mathrm{CO}_{3} \cdot 2 \mathrm{H}_{2} \mathrm{O}$, respectively. Furthermore, the hydration of expansive agent at $60 \%$ is studied in a drying room, which is a standard test environment for measuring the drying shrinkage of concrete in China. The thickness of expansive agent powder is less than $2 \mathrm{~mm}$. After reaching a certain age, the expansive agent was immersed in alcohol for $1 \mathrm{~h}$ to terminate hydration. After immersion was concluded, the sample was vacuum dried for $2 \mathrm{~h}$ and then dried for $1 \mathrm{~d}$ at $105^{\circ} \mathrm{C}$. Finally, the weight loss of the sample during $105^{\circ} \mathrm{C}$ to $1050{ }^{\circ} \mathrm{C}$ was measured by a differential scanning calorimetry (SDTQ600, TA Instruments, New Castle, DE, USA). The hydration degree of the expansive agent was calculated according to Equation (1).

$$
\alpha=\frac{L_{E} \cdot M_{E}}{M_{H} \cdot C}
$$

where $\alpha$ is the hydration degree of $\mathrm{CaO}$ or $\mathrm{MgO}, L_{E}$ is mass loss of the sample during $105^{\circ} \mathrm{C}$ to $1050{ }^{\circ} \mathrm{C}, M_{E}$ is the molar mass of $\mathrm{CaO}$ or $\mathrm{MgO}, M_{H}$ is the molar mass of $\mathrm{H}_{2} \mathrm{O}$, and $\mathrm{C}$ is content of $\mathrm{CaO}$ or $\mathrm{MgO}$ in expansive agent.

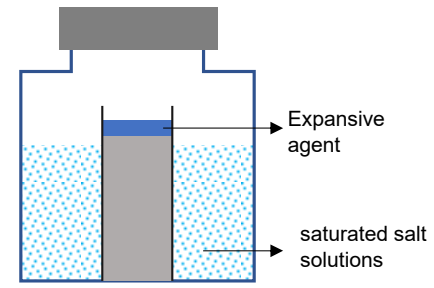

Figure 3. Apparatus for providing a constant $\mathrm{RH}$.

\subsection{Isothermal Calorimetry Measurements}

Hydration process of the paste was determined by isothermal calorimeter (TAM Air Isothermal Calorimeter, TA Instrument). The mixing proportions of pastes were derived from their corresponding concrete mixtures (Table 2) by subtracting the aggregate fraction.

\subsection{Micrographs}

Micrographs of the expansive agent before and after hydration were examined by scanning electron microscope (SEM, FEI).

\section{Results and Discussion}

\subsection{Effect of Expansive Agent on Autogenous Shrinkage of UHPC}

Figure 4 shows the autogenous shrinkage UHPC with and without expansive agent. The results show that $28 \mathrm{~d}$ autogenous shrinkage of UHPC without expansive agent exceeds $600 \mu \varepsilon$, although the coarse aggregate at a volume ratio of 0.17 is added to the 
UHPC. Meanwhile, the autogenous shrinkage at $1 \mathrm{~d}$ and $7 \mathrm{~d}$ reaches $46.7 \%$ and $84.1 \%$ of $28 \mathrm{~d}$, respectively. This ratio shows that the autogenous shrinkage of UHPC develops rapidly in the first few days, and the expansion of the expansive agent before $7 \mathrm{~d}$ may be an effective way to reduce the autogenous shrinkage of UHPC.

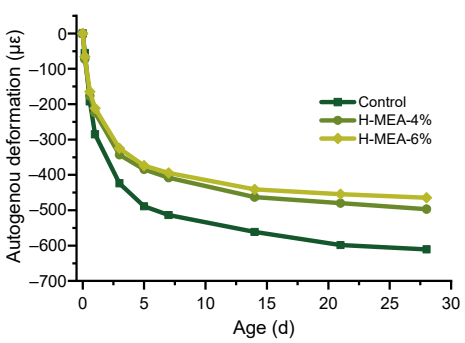

(a)

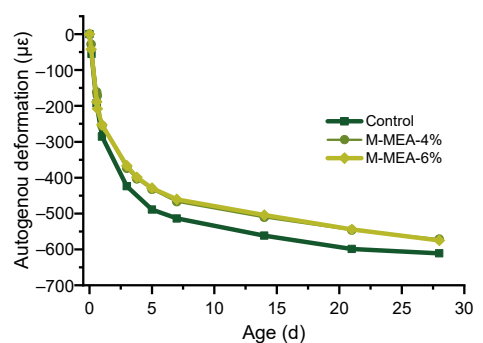

(b)

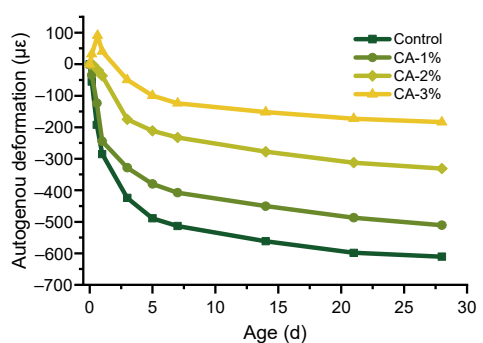

(c)

Figure 4. Autogenous shrinkage of UHPC with and without expansive agent: (a) highly reactive $\mathrm{MgO}$-based expansive agent; (b) medium reactive $\mathrm{MgO}$-based expansive agent; and (c) CaO-based expansive agent.

Figure 4a shows the expansive efficiency of the highly reactive $\mathrm{MgO}$-based expansive agent. The results show that $4 \%$ and $6 \%$ highly reactive $\mathrm{MgO}$-based expansive agents reduce the autogenous shrinkage by $20.5 \%$ and $23.2 \%$ at $7 \mathrm{~d}$, respectively, and $18.6 \%$ and $23.9 \%$ at $28 \mathrm{~d}$, respectively. The results further indicate that expansive efficiency of highly reactive $\mathrm{MgO}$-based expansive agent is very close at $7 \mathrm{~d}$ and $28 \mathrm{~d}$. Figure $4 \mathrm{~b}$ shows that at $28 \mathrm{~d}, 6 \%$ medium reactive $\mathrm{MgO}$-based expansive agent only reduces the autogenous shrinkage by about $5.9 \%$, and there is no significant difference in expansive efficiency between $4 \%$ and $6 \%$ dosage of medium reactive $\mathrm{MgO}$-based expansive agent.

As shown in Figure 4c, the CaO-based expansive agent shows a higher expansive efficiency compared with the MgO-based expansive agent. 1\%, $2 \%$ and $3 \%$ CaO-based expansive agent can reduce the autogenous shrinkage by $16.4 \%, 45.8 \%$ and $70.0 \%$, respectively. In addition, the results show that the $\mathrm{CaO}$-based expansive agent shows an obvious expansion before $1 \mathrm{~d}$, especially in the UHPC with $3.0 \% \mathrm{CaO}$-based expansive agent. It should be noted that in this study, $\mathrm{CaO}$-based expansive agent shows remarkably expansive efficiency at a low mass dosage of $1-3 \%$, while in studies of Shen et al. [10], the mass dosage of $\mathrm{CaO}-$ based expansive agent reached $15 \%$. This difference in the dosage may mainly due to the fact that the $\mathrm{CaO}$-based expansive agent used in this study is grinded from the clinker, while the commercial $\mathrm{CaO}$-based expansive agent generally add other components.

\subsection{Internal $R H$ of $U H P C$}

Figure 5 shows the development of internal RH of the UHPC without expansive agent (Control). At the ages of 3, 7, and $28 \mathrm{~d}$, internal RH of the UHPC is $89.7 \%, 77.0 \%$ and $67.1 \%$, respectively. The test results are consistent with the results of Loukili et al. [24]. In addition, the development process of internal RH is similar to that of the autogenous shrinkage. They all show a rapidly change process in the first few days. Furthermore, the results imply that the expansive agent should have a high hydration activity in the rapidly decreasing process of the $\mathrm{RH}$, especially when the $\mathrm{RH}$ is below $80 \%$, in order to decrease autogenous shrinkage of UHPC effectively. 


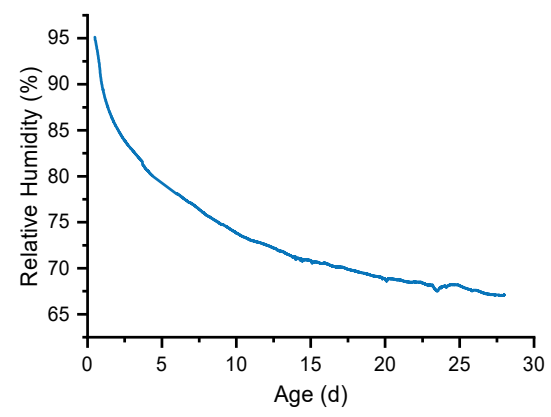

Figure 5. Internal RH of the UHPC.

\subsection{Hydration Degree of Expansive Agent at Different RH}

Figure 6 shows the hydration process of highly reactive $\mathrm{MgO}$-based, medium reactive $\mathrm{MgO}-$ based and $\mathrm{CaO}-$ based expansive agents at $44-93 \% \mathrm{RH}$. The results show that the hydration degree of the three expansive agents decreased with the decrease of $\mathrm{RH}$ at the same age. However, the three expansive agents show significant differences in the humidity sensitivity. As shown in Figure 6a, the $28 \mathrm{~d}$ hydration degree of highly reactive MgO-based expansive agent at RH of $60.0 \%, 81.0 \%$, and $93.0 \%$ is $50.9 \%, 84.1 \%$, and $91.5 \%$, respectively. The results indicate that highly reactive $\mathrm{MgO}$-based expansive agent has hydration activity at RH below $80 \%$. Figure $6 \mathrm{~b}$ shows that at $81.0 \% \mathrm{RH}$, the $28 \mathrm{~d}$ hydration degree of medium reactive $\mathrm{MgO}$-based expansive agent is $25.1 \%$, which is $29.8 \%$ of that of highly reactive $\mathrm{MgO}$-based expansive agent at the same age. Meanwhile, when the $\mathrm{RH}$ decreases to $60 \%$, the 28 d hydration degree of medium reactive $\mathrm{MgO}$-based expansive agent does not exceed $5 \%$. The results indicate that medium reactive $\mathrm{MgO}$-based expansive agent has a low hydration activity at $80 \% \mathrm{RH}$ and has a negligible hydration activity when the $\mathrm{RH}$ decreases to $60 \%$. As shown in Figure $6 \mathrm{c}$, CaO-based expansive agent shows a higher hydration activity at $44.0-93.0 \% \mathrm{RH}$ compared to MgO-based expansive agent used in this study. The $28 \mathrm{~d}$ hydration degree of CaO-based expansive agent reaches $75.0 \%$ at $44 \% \mathrm{RH}$. In addition, the differences of hydration degree of $\mathrm{CaO}$-based expansive agent at $81.0 \%$ and $93.0 \% \mathrm{RH}$ at different ages are less than $15 \%$.

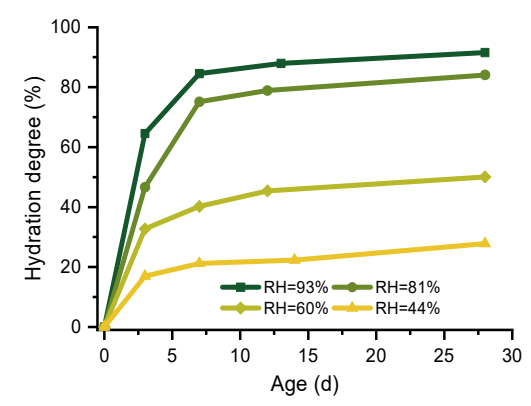

(a)

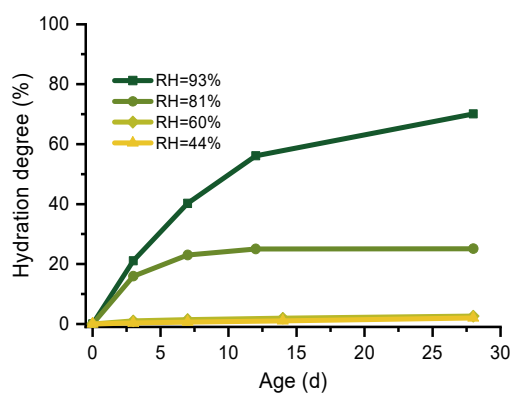

(b)

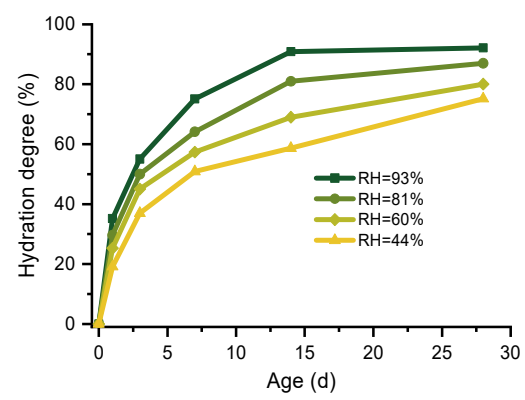

(c)

Figure 6. Hydration degree of expansive agent at different $\mathrm{RH}$ : (a) highly reactive MgO-based expansive agent; (b) medium reactive $\mathrm{MgO}$-based expansive agent; and (c) CaO-based expansive agent.

Consequently, in this work, CaO-based expansive agent has the lowest humidity sensitivity, followed by highly reactive $\mathrm{MgO}$-based expansive agent, and medium reactive $\mathrm{MgO}$-based expansive agent has the highest humidity sensitivity. Furthermore, the differences of expansive efficiency of the three expansive agents in Figure 4 may be explained as follows: The expansive efficiency of CaO-based expansive agent increases with the increase of the dosage, due to its low humidity sensitivity of the hydration. Owing to a high humidity sensitivity of the hydration, medium reactive MgO-based expansive 
agent can hardly hydrate with water in UHPC, especially when the $\mathrm{RH}$ is below $80 \%$, and resulting in a negligible difference in expansive efficiency between $4 \%$ and $6 \%$ dosage. Furthermore, the effectiveness of highly reactive $\mathrm{MgO}$-based expansive agent in UHPC is between $\mathrm{CaO}$-based and medium reactive $\mathrm{MgO}$-based expansive agent.

\subsection{Hydration Heat of the Paste}

The hydration heat of the paste is shown in Figure 7 . The results show that $7 \mathrm{~d}$ hydration heat of the paste with expansive agent is higher than that of the paste without expansive agent. The effect of expansive agent on the hydration heat is mainly due to the higher hydration heat of $\mathrm{CaO}$ and $\mathrm{MgO}$ in expansive agent than that of cement. The results indicate that hydration reaction of the three kind of expansive agents occurs in the first 7 days. The results here can be supported by the results in Figures 5 and 6, which show that in the first few days, when the Internal RH of UHPC is higher than $80 \%$, the hydration reaction of the three expansive agents can occur. In addition, the hydration heat of the paste with $\mathrm{CaO}$-based expansive agent increases significantly with the increase of the content of $\mathrm{CaO}$-based expansive agent, while the difference of hydration heat between $4 \%$ and $6 \%$ medium reactive $\mathrm{MgO}$-based expansive agent is very small. Furthermore, it can be inferred from the results of the hydration heat that $\mathrm{CaO}$-based expansive agent has the highest hydration activity in UHPC paste, when compared with the two kinds of $\mathrm{MgO}$-based expansive agents. This inference is consistent with the results of the humidity sensitivity of the three kinds of expansive agents.

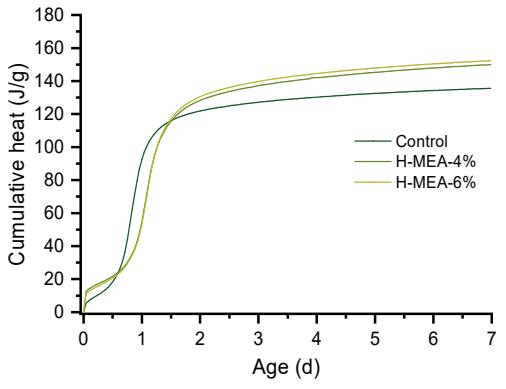

(a)

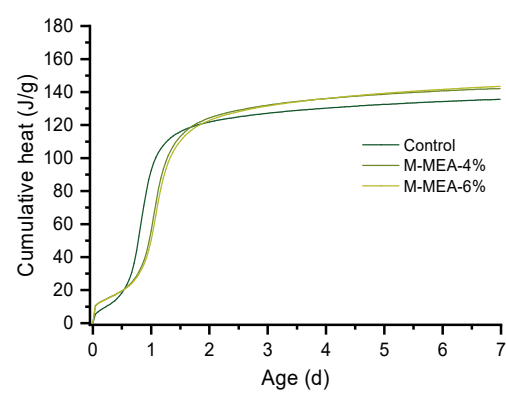

(b)

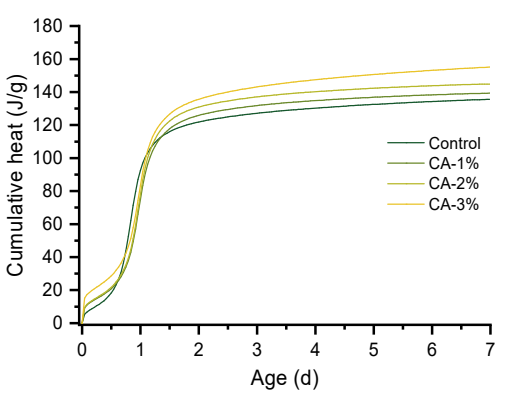

(c)

Figure 7. Hydration heat of the paste with and without expansive agent: (a) highly reactive MgObased expansive agent; (b) medium reactive $\mathrm{MgO}$-based expansive agent; and (c) CaO-based expansive agent.

\subsection{Micrographs Analysis}

Figure 8 shows SEM photographs of three kinds of expansive agents used in this study. A single particle of the MgO-based expansive agent is a porous material and is composed by aggregated magnesia grains [20], which can be clearly observed in Figure $8 \mathrm{~b}$. It should be noted that in highly reactive $\mathrm{MgO}$-based expansive agent, it is difficult to observe the magnesia grains due to their small size (see Figure 8a). During hydration and expansion process of $\mathrm{MgO}$-based expansive agent, the pores of the particle need to be filled by hydration products, and lead to the loss of the expansion efficiency. The porosity of the $\mathrm{MgO}$-based expansive agent may partly explain why the expansion efficiency of $6 \%$ highly reactive $\mathrm{MgO}$-based expansive agent is much lower than that of the $3 \% \mathrm{CaO}$ based expansive agent, although at $60-93 \% \mathrm{RH}$, the differences of the hydration degree between highly reactive $\mathrm{MgO}$-based and $\mathrm{CaO}$-based expansive agent are less than $40 \%$ at different ages. 


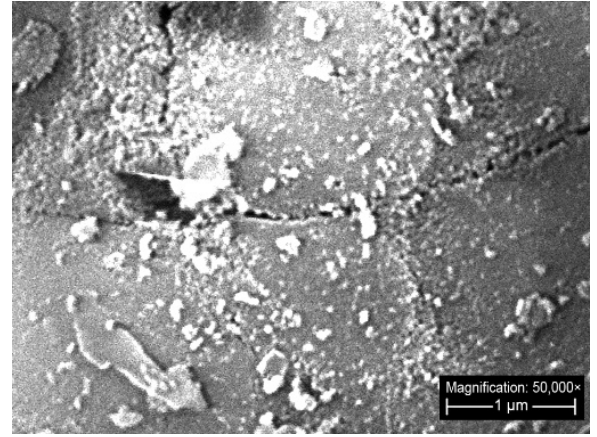

(a)

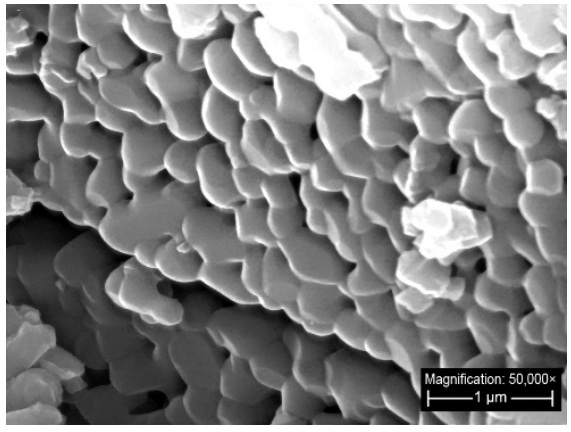

(b)

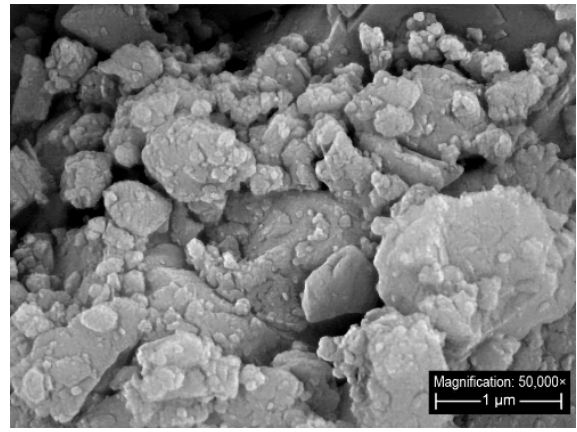

(c)

Figure 8. SEM images of the expansive agents: (a) highly reactive MgO-based expansive agent; (b) medium reactive $\mathrm{MgO}$-based expansive agent; and (c) $\mathrm{CaO}$-based expansive agent.

Figure 9 shows the photograph of the particle of highly reactive $\mathrm{MgO}$-based at $60 \% \mathrm{RH}$. It can be deduced that hydration product- $\mathrm{Mg}(\mathrm{OH})_{2}$ forms in the particle of highly reactive $\mathrm{MgO}-$ based expansive agent from its rougher surface compared to Figure 6a, although the hydration products of $\mathrm{MgO}-$ based expansive agent can be hardly identified by the SEM photograph duo to very small size of the magnesia grains. Unlike MgO-based expansive agent, the hydration of CaO-based expansive agent can be easily observed in photos. When comparing Figures 1 and 10a, it can be observed that the surface shell of clinker of the CaO-based expansive agent is broken due to expansive hydration products at $60 \% \mathrm{RH}$. Furthermore, Figure 10b shows that the formation of sheet hydration products, which is a typical feature of $\mathrm{Ca}(\mathrm{OH})_{2}$. Consequently, the micrographs further confirm that $\mathrm{CaO}$-based and highly reactive $\mathrm{MgO}$-based expansive agents, especially the CaO-based expansive agent, have the hydration activity at RH of above $60 \%$. Furthermore, the results suggest that $\mathrm{CaO}$-based expansive agent is a more effective method to compensate autogenous shrinkage of UHPC than MgO-based expansive agent.

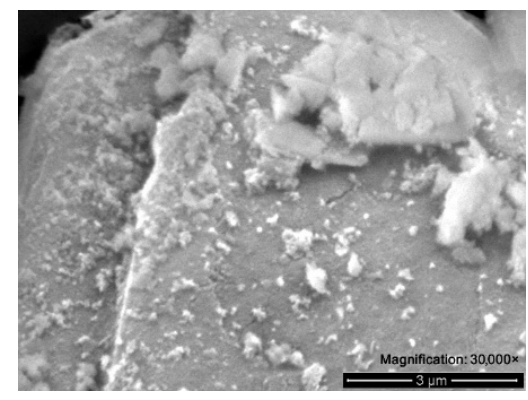

Figure 9. SEM images of highly reactive $\mathrm{MgO}$-based expansive agent cured at $60 \% \mathrm{RH}$ for $28 \mathrm{~d}$.

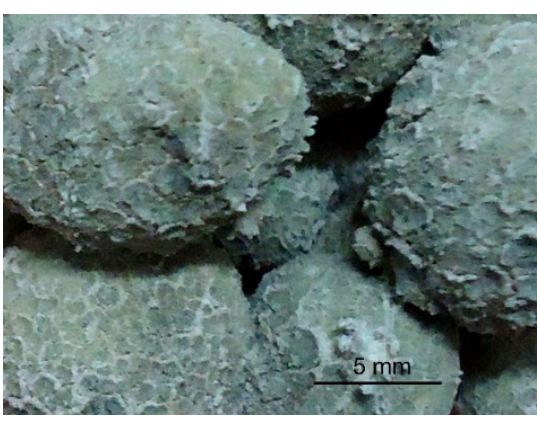

(a)

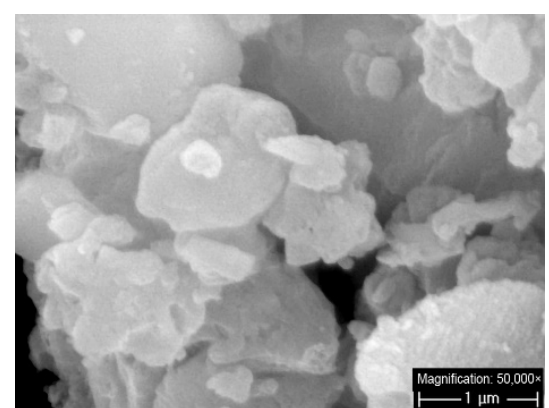

(b)

Figure 10. Images of CaO-based expansive clink/agent cured at $60 \% \mathrm{RH}$ for $28 \mathrm{~d}$ : (a) the clink; and (b) the agent. 


\section{Conclusions}

In this study, highly reactive $\mathrm{MgO}-$ based, medium reactive $\mathrm{MgO}-$ based and $\mathrm{CaO}-$ based expansive agents were used to compensate the autogenous shrinkage of UHPC. The effectiveness of three kinds of expansive agents was investigated through the experimental studies of hydration properties of the expansive agents. The following conclusions can be drawn:

The internal RH of UHPC decreases very quickly, especially in the first few days. The $\mathrm{RH}$ can reduce to below $80 \%$ at $7 \mathrm{~d}$ and below $70 \%$ at $28 \mathrm{~d}$. Under this condition of rapid reduction of the $\mathrm{RH}$, the $\mathrm{CaO}$-based expansive agent has the highest expansive efficiency compared to the two kinds of $\mathrm{MgO}-$ based expansive agents.

The CaO-based expansive agent has a high hydration activity at the $\mathrm{RH}$ of more than $44 \%$. The hydration activity of the two kinds of MgO-based expansive agents decreased significantly with the decrease of the RH. Furthermore, the $28 \mathrm{~d}$ hydration degree of medium reactive $\mathrm{MgO}-$ based expansive agent does not exceed $5 \%$ when the $\mathrm{RH}$ decreases to $60 \%$.

The results indicate that the differences in the expansion efficiency of the three kinds of expansion agents are closely related to their humidity sensitivity, that is, the expansion agent with low humidity sensitivity has high expansion efficiency.

Author Contributions: Y.W. (Yujiang Wang): conceptualization, methodology, investigation, formal analysis, writing—original draft. Q.T.: conceptualization, investigation, writing-review and editing. H.L.: investigation, writing—review and editing. Y.W. (Yang Wang): investigation. All authors have read and agreed to the published version of the manuscript.

Funding: This research was supported by National Natural Science Foundation of China (52008193).

Institutional Review Board Statement: Not applicable.

Informed Consent Statement: Not applicable.

Data Availability Statement: Not applicable.

Conflicts of Interest: The authors declare no conflict of interest.

\section{References}

1. American Society for Testing and Materials (ASTM). ASTM C1856 Standard practice for fabricating and testing specimens of ultra-high performance concrete. In Book of ASTM Standards; American Society for Testing and Materials: Conshohocken, PA, USA, 2017.

2. Shi, C.; Wu, Z.; Xiao, J.; Wang, D.; Huang, Z.; Fang, Z. A review on ultra high performance concrete: Part I. Raw materials and mixture design. Constr. Build. Mater. 2015, 101, 741-751. [CrossRef]

3. Wang, D.; Shi, C.; Wu, Z.; Xiao, J.; Huang, Z.; Fang, Z. A review on ultra high performance concrete: Part II. Hydration, microstructure and properties. Constr. Build. Mater. 2015, 96, 368-377. [CrossRef]

4. Liu, K.; Yu, R.; Shui, Z.; Li, X.; Guo, C.; Yu, B.; Wu, S. Optimization of autogenous shrinkage and microstructure for Ultra-High Performance Concrete (UHPC) based on appropriate application of porous pumice. Constr. Build. Mater. 2019, $214,369-381$. [CrossRef]

5. Koh, K.; Ryu, G.; Kang, S.; Park, J.; Kim, S. Shrinkage properties of ultra-high Performance concrete (UHPC). Adv. Sci. Lett. 2011, 4, 948-952. [CrossRef]

6. Kheir, J.; Klausen, A.; Hammer, T.A.; de Meyst, L.; Hilloulin, B.; van Tittelboom, K.; Loukili, A.; de Belie, N. Early age autogenous shrinkage cracking risk of an ultra-high performance concrete (uhpc) wall: Modelling and Experimental Results. Eng. Fract. Mech. 2021, 257, 108024. [CrossRef]

7. Yang, L.; Shi, C.; Wu, Z. Mitigation techniques for autogenous shrinkage of ultra-high-performance concrete-A review. Compos. Part B Eng. 2019, 178, 107456. [CrossRef]

8. Ghafari, E.; Ghahari, S.A.; Costa, H.; Júlio, E.; Portugal, A.; Durães, L. Effect of supplementary cementitious materials on autogenous shrinkage of ultra-high performance concrete. Constr. Build. Mater. 2016, 127, 43-48. [CrossRef]

9. Valipour, M.; Khayat, K.H. Coupled effect of shrinkage-mitigating admixtures and saturated lightweight sand on shrinkage of UHPC for overlay applications. Constr. Build. Mater. 2018, 184, 320-329. [CrossRef]

10. Shen, P.; Lu, L.; He, Y.; Wang, F.; Lu, J.; Zheng, H.; Hu, S. Investigation on expansion effect of the expansive agents in ultra-high performance concrete. Cem. Concr. Compos. 2020, 105, 103425. [CrossRef] 
11. Li, S.; Mo, L.; Deng, M.; Cheng, S. Mitigation on the autogenous shrinkage of ultra-high performance concrete via using mgo expansive agent. Constr. Build. Mater. 2021, 312, 125422. [CrossRef]

12. Soliman, A.M.; Nehdi, M.L. Effects of shrinkage reducing admixture and wollastonite microfiber on early-age behavior of ultra-high performance concrete. Cem. Concr. Compos. 2014, 46, 81-89. [CrossRef]

13. Justs, J.; Wyrzykowski, M.; Bajare, D.; Lura, P. Internal curing by superabsorbent polymers in ultra-high performance concrete. Cem. Concr. Res. 2015, 76, 82-90. [CrossRef]

14. Liu, J.; Farzadnia, N.; Khayat, K.H.; Shi, C. Effects of SAP characteristics on internal curing of UHPC matrix. Constr. Build. Mater. 2021, 280, 122530. [CrossRef]

15. Liu, J.; Han, F.; Cui, G.; Zhang, Q.; Lv, J.; Zhang, L.; Yang, Z. Combined effect of coarse aggregate and fiber on tensile behavior of ultra-high performance concrete. Constr. Build. Mater. 2016, 121, 310-318. [CrossRef]

16. Yoo, D.Y.; Banthia, N. Mechanical properties of ultra-high-performance fiber-reinforced concrete: A review. Cem. Concr. Compos. 2016, 73, 267-280. [CrossRef]

17. Li, P.P.; Yu, Q.L.; Brouwers, H.J.H. Effect of coarse basalt aggregates on the properties of Ultra-high Performance Concrete (UHPC). Constr. Build. Mater. 2018, 170, 649-659. [CrossRef]

18. Szostak, B.; Golewski, G.L. Rheology of cement pastes with siliceous fly ash and the csh nano-admixture. Materials 2021, 14, 3640. [CrossRef]

19. Hisseine, O.A.; Soliman, N.A.; Tolnai, B.; Tagnit-Hamou, A. Nano-engineered ultra-high performance concrete for controlled autogenous shrinkage using nanocellulose. Cem. Concr. Res. 2020, 137, 106217. [CrossRef]

20. Liu, C.; He, X.; Deng, X.; Wu, Y.; Zheng, Z.; Liu, J.; Hui, D. Application of Nanomaterials in Ultra-High Performance Concrete: A Review. Nanotechnol. Rev. 2020, 9, 1427-1444. [CrossRef]

21. Mehta, P.K. Mechanism of expansion associated with ettringite formation. Cem. Concr. Res. 1973, 3, 1-6. [CrossRef]

22. Li, H.; Tian, Q.; Zhao, H.; Lu, A.; Liu, J. Temperature sensitivity of MgO expansive agent and its application in temperature crack mitigation in shiplock mass concrete. Constr. Build. Mater. 2018, 170, 613-618. [CrossRef]

23. Mo, L.; Deng, M.; Tang, M. Effects of calcination condition on expansion property of MgO-type expansive agent used in cement-based materials. Cem. Concr. Res. 2010, 40, 437-446. [CrossRef]

24. Loukili, A.; Khelidj, A.; Richard, P. Hydration kinetics, change of relative humidity, and autogenous shrinkage of ultra-highstrength concrete. Cem. Concr. Res. 1999, 29, 577-584. [CrossRef]

25. Huang, H.; Ye, G. Examining the "time-zero" of autogenous shrinkage in high/ultra-high performance cement pastes. Cem. Concr. Res. 2017, 97, 107-114. [CrossRef]

26. Mo, L.; Deng, M.; Tang, M.; Al-Tabbaa, A. MgO expansive cement and concrete in China: Past, present and future. Cem. Concr. Res. 2014, 57, 430-436. [CrossRef]

27. Li, H.; Wang, Y.; Wang, Y.; Liu, J.; Tian, Q. Effect of $\mathrm{CaO}$ and $\mathrm{MgO}$ based expansive agent on deformation and mechanical properties of concrete-filled steel tubes. Constr. Build. Mater. 2020, 250, 118723. [CrossRef]

28. American Society for Testing and Materials (ASTM). ASTM C403 Standard test method for time of setting of concrete mixtures by penetration resistance. In Book of ASTM Standards; American Society for Testing and Materials: Conshohocken, PA, USA, 2016. 